\title{
Effect of magnetized variable thermal conductivity on flow and heat transfer characteristics of unsteady Williamson fluid
}

https://doi.org/10.1515/nleng-2020-0020

Received Mar 27, 2020; accepted Jun 9, 2020.

\begin{abstract}
A two-dimensional mathematical model of magnetized unsteady incompressible Williamson fluid flow over a sensor surface with variable thermal conductivity and exterior squeezing with viscous dissipation effect is investigated, numerically. Present flow model is developed based on the considered flow geometry. Effect of Lorentz forces on flow behaviour is described in terms of magnetic field and which is accounted in momentum equation. Influence of variable thermal conductivity on heat transfer is considered in the energy equation. Present investigated problem gives the highly complicated nonlinear, unsteady governing flow equations and which are coupled in nature. Owing to the failure of analytical/direct techniques, the considered physical problem is solved by using Runge-Kutta scheme (RK-4) via similarity transformations approach. Graphs and tables are presented to describe the physical behaviour of various control parameters on flow phenomenon. Temperature boundary layer thickens for the amplifying value of Weissenberg parameter and permeable velocity parameter. Velocity profile decreased for the increasing squeezed flow index and permeable velocity parameter. Increasing magnetic number increases the velocity profile. Magnifying squeezed flow index magnifies the magnitude of Nusselt number. Also, RK4 efficiently solves the highly complicated nonlinear complex equations that are arising in the fluid flow problems. The present results in this article are significantly matching with the published results in the literature.
\end{abstract}

Keywords: Williamson fluid; Weissenberg number; sensor surface; magnetic field; variable thermal conductivity; squeezed flow

Usha Shankar, Department of Mathematics, Gulbarga University, Kalaburagi-585 106, Karnataka, India

Department of Karnataka Power Corporation Limited, Raichur Thermal Power Station, Shaktinagar-584 170, Raichur, Karnataka, India

\section{Introduction}

Due to the substantial development in the modern technology, a keen attention has been given to analyze the heat transfer characteristics of squeezing flows in various geometries. Since, squeezed flows have good number of significant practical and industrial applications in various fields of science and engineering such as, polymer processing, food engineering, injection molding, lubrication systems, formation of foam, blood flow inside the vessels, cooling towers, bi-axial expansion of bubble boundaries, hydro-dynamical machines, compression, moisture migration, chemical engineering, dampers, heating/cooling processes and many others. However, the applied normal stresses to the moving surfaces/plates results the squeezing flow. The detailed literature and applications of squeezing flows can be found in $[1,2]$. Another considerable example of squeezing flow is the movement of diarthrodial joints and valves in the human body and which is associated with the field of mathematical bioengineering and biomedicine [3]. In the present biological and chemical technology, the sensors with stretching surfaces as their sensing elements playing a key role in determination of various diseases, hazardous and bio-warfare elements. In practical, the above mentioned problems are tackled by using a micro-cantilever which bends upon the binding of the target molecules with the receptor coating on one of its surfaces. Clearly, in practical applications, the micro-cantilever is usually positioned in a film of thin fluidic cells with external squeezing disturbance. This physical situation of fluid motion over a micro-cantilever is modeled as flow about a sensor surface. Literature $[4,5]$ gives the detailed study of micro-cantilever, electrochemical, biosensors and their applications in various fields of biomedicine.

\footnotetext{
*Corresponding Author: N. B. Naduvinamani, Department of Mathematics, Gulbarga University, Kalaburagi-585 106, Karnataka, India, E-mail: naduvinamaninb@yahoo.co.in Hussain Basha, Department of Mathematics, Central University of Karnataka, Kalaburagi-585 367, Karnataka, India
} 
However, in the field of engineering sciences, the heat transfer problems have plenty of scientific applications in space cooling, climate engineering, metallurgical processes, laser cooling, magnet and radiative cooling process, cooling of nuclear reactor, petroleum industries, conduction of heat in tissues, thermal energy storage and many others advantages. In this direction, Stefan [6] made considerable contribution to the field of squeezing flows by developing a mathematical model. Later, number of investigators continued the Stefan's problem by considering the different geometries with suitable modifications. Kuzma [7] investigated the influence of inertia effects on the squeeze film generated between two parallel plates. Also, literature [7] gives the experimental validation of the theoretical results. The squeezing flow between two parallel plates with full unsteady Navier-Stokes in terms of radial and rectilinear flow was studied by Gupta and Gupta [8]. It is observed from their investigation that, the obtained self-similar solutions holds when the length between the plates changes as a square root of linear function of time. Wang and Watson [9] investigated the thermodynamic behaviour of squeezed flow between two elliptic parallel plates by assuming the length between plates change as inverse square root of time. Further, the literature [9] modeled the two-point boundary value problem and which is being solved by using suitable mathematical techniques such as, perturbation scheme and homotopy analysis method (HAM). Their study shows that, for higher values of squeezed number, there occurs a boundary layer on the plates with negligible viscosity. Usha and Sridharan [10] numerically investigated the arbitrary squeezed flow of viscous incompressible fluid flow in a channel with time-dependent length between two elliptic parallel plates. Their study utilizes the multifold series method to solve the governing fluid flow equations and it is remarked from their analysis that, the magnifying Reynolds number upsurge the fluid motion in the vicinity of plate surface. Bhattacharyya and Pal [11] studied the magnetized squeezing flow of viscous incompressible electrically conducting fluid film generated between two parallel discs. Further, their study assumes that, the lower disc will rotate with transient arbitrary angular velocity. Also, the literature [11] utilizes the standard Hermitian finite difference scheme to generate the numerical solutions. However, it is noticed from their investigation that, the increasing length between plates enhance the load and also the increasing magnetic number and angular velocity amplifies the torque on the lower disc.

The problem of incompressible rectilinear timedependent, two-dimensional magnetized viscous squeezed flow through an infinite channel with ho- motopy analysis technique was investigated by Siddiqui et al. [12]. Their study shows that, decaying magnetic field describes the viscous behaviour of considered fluid. Rashidi et al. [13] studied the analytical solution of the squeezing flow between circular plates by semi-numerical technique such as homotopy analysis method. Further, the enhanced Reynolds number shows the cross flow behaviour on axial velocity profile. The problem of incompressible transient viscous squeezed flow of twodimensional fluid between two parallel plates under the impact of chemical reaction and viscous dissipation was investigated by Mustafa et al. [14] through HAM. Literature [14] depicts that the magnifying squeezing number diminishes the concentration field and increases the momentum transport coefficient. Dogonchi and Ganji [15] analyzed the impact of Brownian motion on radiative squeezing flow of nanofluid between two non-parallel stretching surfaces under the steady-state condition with heat source/sink. Their investigation depicts that, temperature profile decreases with enhancing radiation parameter. Hayat et al. [16] demonstrated the magnetized time-dependent, two-dimensional, incompressible, couple stress nanofluid flow between two parallel plates with chemical reaction effects. Their study shows that, the increasing squeezing flow parameter diminish the thermal field. Also, the axial velocity profile shows the cross flow behaviour with larger magnetic parameter values in the solution regime. Further, the pioneering work in this direction can be found in the literature [17-21].

Due to the immense development in the bioengineering technology, the magnetic flows attracted the interest of many investigators because of their wide-spread applications. Also, the flow and heat transfer characteristics are tuned according to the requirement by applying the magnetic field. In the modern biomedicine field, the principles of MHD (magneto-hydrodynamic) are strongly employed to cure the tedious pathological situations. Because of these reasons, the concept of MHD has good practical advantageous in the present days. The theoretical and experimental investigation to explore the effect of magnetic number on lubrication flows between two parallel plates was studied by Maki et al. [22]. Further, their investigation shows that the effects of fluid inertia on flow behaviour. Also, their study demonstrates the validation of theoretical results with the experimental ones. The magnetized three dimensional bidirectional flow of nanofluid under steady-state condition with second order slip and chemical reaction effects was studied by Hayat et al. [23]. The semi-analytical investigation in the literature [23] depicts that the amplifying magnetic parameter decay the flow field. Further, the concentration profile enhances for 
the increasing heterogeneous chemical reaction parameter. Khan et al. [24] analyzed the magnetized incompressible viscous squeezed flow between two parallel plates under steady-state condition. Their study shows that the increasing magnetic parameter results the dual behaviour for velocity field. Khan et al. [25] studied the effect of magnetic field on incompressible two-dimensional Casson fluid flow between two parallel plates with HPM scheme. Their investigation shows that the decaying velocity field corresponds to the increasing magnetic parameter.

Shah et al. [26] thermodynamically demonstrated the impact of Lorentz forces on two-dimensional squeezed flow of electrically conducting fluid through a channel under transient condition via homotopy analysis technique. Their investigation shows that, the increasing squeezing number increases the axial velocity field. Uddin et al. [27] studied the transient magnetized of bio-nano fluid flow over a stretching sheet with heat and mass transfer process. Their analysis shows that the enhancing magnetic parameter increases the temperature and concentration profiles. The phenomena of peristaltic heat and mass transport in a channel flow of magneto-hydrodynamic nanofluid under unsteady state condition with nanofluid properties was investigated numerically using HAM by Hayat et al. [28]. Their investigation shows that the enhancing magnetic number diminishes the thermal profile. Dogonchi and Ganji [29] discussed the magnetized unsteady nanofluid flow between two parallel plates using Duan-Rach approach. Their study shows that the enhanced heat generation parameter magnifies the Nusselt number. Akmal et al. [30] described the impact of Lorentz forces on flow and heat transfer behaviour of nanofluid under the squeezing conditions with entropy effect. Their numerical study shows that the enhancing thermal relaxation time parameter decays the entropy generation rate and thermal field in the flow region. The detailed study in this direction can be found in the available literature [3136].

Khaled and Vafai [37] studied the magnetized twodimensional electrically conducting flow behaviour of viscous fluid inside a thin film of channel under the oscillatory squeezing conditions. Their investigation explored that the magnifying Reynolds number corresponds to the enhanced instabilities in the fluid motion. Khaled and Vafai [38] investigated the impact of normal magnetic field on squeezed flow of electrically conducting Newtonian fluid flow about a sensor surface under the external squeezing condition. It is clear from their analysis that, the magnifying Prandtl number decays the thermal profile. Haq et al. [39] studied the two-dimensional mathematical modeling of magnetized squeezed nanofluid flow about a sensor surface with unsteady conditions. Their numerical analysis shows that the increased nanoparticle volume fraction diminishes the temperature field. Khan et al. [40] numerically studied the impact of Lorentz forces on thermal characteristics of squeezed flow of Carreau fluid past a sensing surface with time-dependent conditions with respect to external squeezing via RK-4 technique. Their study remarks that the magnifying Weissenberg number decays the axial velocity profile. The timedependent mathematical model of magnetized electrically conducting squeezed flow of Carreau-Yasuda fluid about a sensor surface with external squeezing was studied by Salahuddin et al. [41] using RKF technique. Their study explored that the increasing permeable velocity parameter decays the local momentum transport coefficient. The impact of transverse magnetic field on squeezed hyperbolic tangent fluid and its thermal flow behaviour about a sensor surface under external squeezing with unsteady conditions was investigated by Kumar et al. [42] via RKF scheme. Their analysis shows that the enhanced squeezed flow index suppresses the velocity profile.

Ahmad et al. [43] studied the effect of CattaneoChristov heat flux model on the stagnation point flow of micropolar nanofluid over a stretching surface with slip effects for single-wall carbon nanotube and multi-wall carbon nanotube. It is observed from their analysis that, the Bejan number is a decreasing function of Brinkman parameter in the flow region. Ramesh et al. [44] investigated the flow and heat transfer characteristics of Sakiadis and Blasius flow of Williamson fluid with convective boundary condition over a flat plate. It is noticed from their analysis that, the Blasius flow gives the thicker temperature boundary layer when compared to the Sakiadis flow. Usha et al. [45] numerically analyzed the impacts of CattaneoChristov double diffusion heat and mass flux models on the transient nanofluid flow between two parallel plates with Joule heating and chemical reaction. It is remarked from their study that, the concentration profile is a decaying function of thermophoresis parameter and increasing function of Brownian motion parameter. Mishra et al. [46] demonstrated the effect of non-uniform heat source on magnetized power-law fluid flow over a stretching sheet with non-Darcian porous medium. Their investigation illustrated that, the power-law fluid shows a dual property in the presence of applied magnetic field.

Authors have motivated by the above cited literature review and advantageous of squeezing flow in various branches of science and engineering including biomedicine and biology. In view of this reason authors have made an attempt to investigate the flow and heat transfer behaviour of unsteady Williamson fluid over a 
horizontal sensor surface under the external squeezing with transverse magnetic field. The present literature review witnessed that, the current problem is of very much engineering interest. Based on the present literature review, the present investigation is yet to be report. Further, the current physical situation in this article is mathematically devised by utilizing considered flow configuration and resulting nonlinear system was solved by employing RK-4 technique. Also, the self-similar non-dimensional numerical solutions are presented with the help of graphs and tables. Finally, the accuracy of present results is accounted.

\section{Williamson fluid model}

For the flow of viscous incompressible Williamson fluid [47-50], the governing equations of continuity and momentum are given as follows:

$$
\begin{gathered}
\operatorname{div} \mathbf{V}=0 \\
\rho\left(\frac{d V}{d t}\right)=\operatorname{div} \mathbf{S}+\rho \mathbf{c}
\end{gathered}
$$

In the above Eqs. (1) and (2), $\mathbf{V}$ be the velocity vector, $\rho$ indicate the density, Cauchy stress tensor is denoted with $\mathbf{S}$, body force vector is denoted with $\mathbf{c}$ and $\left(\frac{d}{d t}\right)$ indicate the material derivative with respect to time. The governing Cauchy stress tensor and Williamson fluid flow equations are obtained as follows:

$$
\begin{gathered}
\mathbf{S}=-p \mathbf{I}+\tau \\
\tau=\left[\mu_{\infty}+\frac{\mu_{o}-\mu_{\infty}}{1-\Gamma \dot{Y}}\right] \mathbf{A}_{1}
\end{gathered}
$$

In the above Eqs. (3) and (4), $p$ be the pressure, I indicate the identity vector, $\tau$ be the extra stress tensor, $\mu_{o}$ and $\mu_{\infty}$ are the zero and infinite shear rate viscosities, time constant is denoted with $\Gamma>0$ and $\mathbf{A}_{1}$ be the Rivlin-Erickson first tensor. Further, the symbol $\dot{Y}$ is determined as below:

$$
\begin{gathered}
\dot{Y}=\sqrt{\frac{1}{2} \pi} \text { where, } \pi=\operatorname{trace}\left(\mathbf{A}_{1}\right)^{2} \\
\dot{Y}=\left[2\left(\frac{\partial u}{\partial x}\right)^{2}+\left(\frac{\partial u}{\partial y}+\frac{\partial v}{\partial x}\right)^{2}+2\left(\frac{\partial v}{\partial y}\right)^{2}\right]^{\frac{1}{2}}
\end{gathered}
$$

In the above Eq. (5), the second invariant strain tensor is denoted with $\pi$. Also, $\mu_{\infty}=0$ and $\Gamma \dot{Y}<1$ be the cases considered in the present paper. With these assumptions, the tensor defined in Eq. (4) takes following form:

$$
\tau=\left[\frac{\mu_{o}}{1-\Gamma \dot{Y}}\right] \mathbf{A}_{1}
$$

With the help of binomial expansion, the Eq. (7) is written as follows:

$$
\tau=\mu_{o}[1+\Gamma \dot{Y}] \mathbf{A}_{1}
$$

Thus, following are the components of extra stress tensor $\tau$.

$$
\begin{aligned}
& \tau_{x x}=2 \mu_{o} \\
& \left(1+\Gamma\left\{\left[2\left(\frac{\partial u}{\partial x}\right)^{2}+\left(\frac{\partial u}{\partial y}+\frac{\partial v}{\partial x}\right)^{2}+2\left(\frac{\partial v}{\partial y}\right)^{2}\right]^{\frac{1}{2}}\right\}\right)\left(\frac{\partial u}{\partial x}\right), \\
& \tau_{x y}=\tau_{y x}=\mu_{o} \\
& \left(1+\Gamma\left\{\left[2\left(\frac{\partial u}{\partial x}\right)^{2}+\left(\frac{\partial u}{\partial y}+\frac{\partial v}{\partial x}\right)^{2}+2\left(\frac{\partial v}{\partial y}\right)^{2}\right]^{\frac{1}{2}}\right\}\right)\left(\frac{\partial u}{\partial y}+\frac{\partial v}{\partial x}\right), \\
& \tau_{y y}=2 \mu_{o} \\
& \left(1+\Gamma\left\{\left[2\left(\frac{\partial u}{\partial x}\right)^{2}+\left(\frac{\partial u}{\partial y}+\frac{\partial v}{\partial x}\right)^{2}+2\left(\frac{\partial v}{\partial y}\right)^{2}\right]^{\frac{1}{2}}\right\}\right)\left(\frac{\partial v}{\partial y}\right) .
\end{aligned}
$$

\section{Mathematical formulation}

A two-dimensional mathematical model of magnetized time-dependent, viscous incompressible, electrically conducting Williamson fluid flow about a sensor surface with variable thermal conductivity and exterior squeezing with viscous dissipation effect is investigated, numerically. Figure 1 illustrates the flow configuration of the present problem (closed squeezed channel) with all necessary conditions. Also, $h(t)$ be the time-dependent height of the closed channel taken as 0 to $h$ and which is much larger than boundary layer thickness. Further, the microcantilever sensor of length $L$ is enclosed inside the channel and the upper surface of the channel is squeezed but the lower surface is fixed. However, it is quite obvious to assume that, the squeezing process is presumed to be initiates from the tip of the sensor surface to the free stream fluid. The fluid flow is driven by the external free stream velocity $U(x, t)$ and the magnetic field of strength $B_{o}$ is applied normal to the channel. Further, the thermodynamic behaviour of current physical situation is tackled with the help of well-established rectangular coordinate system in which $x$-coordinate is taken along axial direction and $y$-axis is chosen normal to $x$-direction. Thus, by making the use of above assumptions and by considering the Williamson fluid model as described in the Section 2 
along with $\tau_{x z}=\tau_{y z}=\tau_{z x}=\tau_{z y}=\tau_{z z}=0$, laws of fluid motion are defined as follows [38-42, 47-50].

Equation of continuity:

$$
\frac{\partial u}{\partial x}+\frac{\partial v}{\partial y}=0
$$

Momentum equation:

$$
\begin{aligned}
& \frac{\partial u}{\partial t}+u \frac{\partial u}{\partial x}+v \frac{\partial u}{\partial y}=-\frac{1}{\rho}\left(\frac{\partial p}{\partial x}\right)+v \frac{\partial^{2} u}{\partial y^{2}}+2 v \Gamma \frac{\partial u}{\partial y} \frac{\partial^{2} u}{\partial y^{2}} \\
& -\left(\frac{\sigma B_{o}^{2}}{\rho}\right) u
\end{aligned}
$$

Free stream equation:

$$
\frac{\partial U}{\partial t}+U \frac{\partial U}{\partial x}=-\frac{1}{\rho}\left(\frac{\partial p}{\partial x}\right)-\left(\frac{\sigma B_{o}^{2}}{\rho}\right) U
$$

Energy equation:

$$
\begin{aligned}
& \frac{\partial T}{\partial t}+u \frac{\partial T}{\partial x}+v \frac{\partial T}{\partial y}=\frac{\partial}{\partial y}\left(\alpha(T) \frac{\partial T}{\partial y}\right) \\
& +\frac{\mu}{\rho C_{p}}\left(1+\Gamma \frac{\partial u}{\partial y}\right)\left(\frac{\partial u}{\partial y}\right)^{2}
\end{aligned}
$$

In the above Eqs. (10)-(13), $u$ and $v$, be the velocity components along $x$ and $y$ directions, free stream velocity along axial flow path is described with $U$, fluid temperature denoted with $T, t$ be the time, working fluid density is denoted with $\rho, \Gamma$ be the time constant, $\alpha(T)$ be the variable thermal conductivity, $v$ be the kinematic viscosity of the working liquid and $B_{o}$ be the magnetic strength. Also, Eqs. (11) and (13) satisfies the necessary flow conditions within the boundary layer region considered for the present study. Further, Eq. (12) governs the outer free stream flow ( $u \rightarrow U, v \rightarrow 0$ ) which is assumed to be invisid and uniform with respect to the normal coordinate. However, error in the above prediction was eradicated by considering the small sensor length in accordance with channel height [38]. Finally, the following required flow equation is obtained by eliminating the pressure term in Eq. (11) using Eq. (12) as

$$
\begin{aligned}
& \frac{\partial u}{\partial t}+u \frac{\partial u}{\partial x}+v \frac{\partial u}{\partial y}=\frac{\partial U}{\partial t}+U \frac{\partial U}{\partial x}+v \frac{\partial^{2} u}{\partial y^{2}} \\
& +2 v \Gamma \frac{\partial u}{\partial y} \frac{\partial^{2} u}{\partial y^{2}}+\frac{\sigma B_{o}^{2}}{\rho}(U-u)
\end{aligned}
$$

Thus, the considered flow equations Eqs. (13) and (14) are simplified with respect to hydromagnetic \& thermal conditions as listed below [38-42].

$$
\begin{aligned}
& u(x, 0, t)=0, \quad v(x, 0, t)=v_{o}(t), \\
& -k \frac{\partial T(x, 0, t)}{\partial y}=q(x) \text { at } y=0 \\
& u(x, \infty, t)=U(x, t), T(x, \infty, t)=T_{\infty} \text { at } y=\infty
\end{aligned}
$$

The symbols used in the Eq. (15) are defined as, $U(x, t), T_{\infty}$ be the ambient fluid velocity and temperature, the surface heat flux is indicated with $q(x)$. Further, in the present case, the variable thermal conductivity $\alpha(T)$ is expressed as $\alpha(T)=\alpha_{\infty}(1+\epsilon \theta)$, where $\epsilon$ be the small quantity. Suppose that, if sensor surface behaves as a function surface heat flux $q(x)$, then $v_{o}(t)$ describe the reference velocity adjacent to the surface when wall is considered to be permeable. However, by making the use of appropriate similarity transformations, the set of coupled two-dimensional transient Williamson fluid flow Eqs. (13) and (14) with necessary conditions Eq. (15) are transformed to set of nonlinear ordinary differential equations. Thus, to achieve this objective the following similarity transformations are used.

$$
\begin{aligned}
& U=a x, \quad u=a x f^{\prime}(\eta), \quad \eta=y \sqrt{\frac{a}{v}}, \quad \psi=f(\eta) x \sqrt{a v}, \\
& a=\frac{1}{s+b t} \quad v=-f(\eta) \sqrt{a v}, \quad \theta(\eta)=\frac{T-T_{\infty}}{\frac{q_{o} x}{k} \sqrt{\frac{v}{a}}}, \\
& v_{o}(t)=v_{i} \sqrt{a}, \quad q(x)=q_{o} x
\end{aligned}
$$

In the above Eq. (16), $s$ be the arbitrary constant, squeezed flow strength is denoted with $a, q_{o}$ be the surface heat flux and $k$ be the thermal conductivity. Further, the motion of channel height satisfies the equation $h(t)=$ $\frac{1}{(s+b t)^{\frac{1}{b}}}$ when $b>0$ and $h(t)=h_{o} e^{-s t}$ when $b=0, h_{o}$ be the constant [38]. Also, it is quite obvious that, the velocity $f_{o}$ enhanced for decaying time $t$ with $b>0$, since, the squeezing velocities magnifies for the decreasing time $t$ within the boundary layer region. However, $\psi$ holds continuity relation with $u=\frac{\partial \psi}{\partial y}$ and $v=-\frac{\partial \psi}{\partial x}$. Finally, the substitution of Eq. (16), into the Eqs. (13)-(15) gives the following nondimensional flow system in view of $\eta$.

$$
\left.\begin{array}{l}
f^{\prime \prime \prime}(\eta)+\left(f(\eta)+\frac{b \eta}{2}\right) f^{\prime \prime}(\eta)-\left(f^{\prime}(\eta)\right)^{2} \\
+2 W e f^{\prime \prime}(\eta) f^{\prime \prime \prime}(\eta)+M\left(1-f^{\prime}(\eta)\right)+b\left(f^{\prime}(\eta)-1\right) \\
+1=0
\end{array}\right\}
$$

$$
\left.\begin{array}{l}
(1+\epsilon \theta(\eta)) \theta^{\prime \prime}(\eta)+\operatorname{Pr}\left(f(\eta)+\frac{b \eta}{2}\right) \theta^{\prime}(\eta) \\
-\operatorname{Pr}\left(f^{\prime}(\eta)+\frac{b}{2}\right) \theta(\eta)+\epsilon\left(\theta^{\prime}(\eta)\right)^{2}+\operatorname{PrEc}\left(f^{\prime \prime}(\eta)\right)^{2} \\
+\operatorname{PrEcWe}\left(f^{\prime \prime}(\eta)\right)^{3}
\end{array}\right\}
$$

Following are the transformed boundary conditions with respect to $\eta$.

$$
\left.\begin{array}{rl}
f(0)=-f_{o}, & f^{\prime}(0)=0, \theta^{\prime}(0)=-1 \text { at } \eta=0 \\
f^{\prime}(\infty)=1 & \theta(\infty)=0 \text { at } \eta=\infty
\end{array}\right\}
$$

In the above Eq. (17)-(19), the superscript "prime" portray the derivative with respect to $\eta$. Further, the governing 
physical parameters controlling the fluid flow are defined as follows.

$$
M=\frac{\sigma B_{o}^{2}}{\rho a}
$$

(Magnetic number), $W e=\Gamma U \sqrt{\frac{a}{v}}$ (Weissenberg number), $b$ (squeezed flow index), $f_{o}=\frac{v_{i}}{\sqrt{v}}$ (permeable velocity parameter), $\operatorname{Pr}=\frac{v}{\alpha}$ (Prandtl number), $\epsilon$ (small parameter) and $E c=\frac{U^{2}}{C_{p}\left(\frac{q_{0}}{k}\right) \sqrt{\frac{v}{a}}}$ (Eckert number).

The Prandtl number is an example of a dimensionless number that is an intrinsic property of a fluid. Fluids with small Prandtl numbers are free-flowing liquids with high thermal conductivity and are therefore a good choice for heat conducting liquids. It is clear that, the liquid metals are very good heat transfer liquids. Interestingly, air is a decent heat transfer liquid as well, whereas typical organic solvents are not. With increasing viscosity, the momentum transport dominates over the heat transport, which makes these liquids a bad choice for heat conduction. Hence, Prandtl number is taken to be small for non-Newtonian fluids. The Eckert number is used to characterize the influence of self-heating of a fluid as a consequence of dissipation effects.

The engineering quantities of interest namely, skinfriction coefficient and heat transfer rate contributes significant outcomes in many of the technical applications. Hence, we have calculated computer generated numerical values for skin-friction coefficient and heat transfer rate in this paper and which are tabulated in Table 1 and Figures 13-15. However, with the help of defined thermodynamic conditions, the wall shear stress and heat transfer rate are given below.

$$
\begin{gathered}
C_{f}=\frac{2 \tau_{w}}{\rho U^{2}} \\
N u_{x}=\frac{x q_{w}}{q_{o} x \sqrt{\frac{v}{a}}}
\end{gathered}
$$

In the above Eqs. (20) and (21), the values of $\tau_{w}$ and $q_{w}$ are obtained as follows:

$$
\tau_{w}=\mu_{o}\left(\frac{\partial u}{\partial y}+\Gamma\left(\frac{\partial u}{\partial y}\right)^{2}\right)_{y=0} \text { and } q_{w}=-k\left(\frac{\partial T}{\partial y}\right)_{y=0}
$$

Finally, in view of similarity variable $\eta$, the equations of skin-friction coefficient and Nusselt number are obtained by using the Eqs. (16) and (22) into the Eqs. (20) and (21) as follows:

$$
\begin{gathered}
\frac{1}{2} C_{f} \sqrt{R e_{x}}=f^{\prime \prime}(0)+W e\left(f^{\prime \prime}(0)\right)^{2} \\
N u_{x}\left(R e_{x}\right)^{\frac{-1}{2}}=-\theta^{\prime}(0)
\end{gathered}
$$

Thus, the Eqs. (23) and (24) are the required equations of skin-friction coefficient and Nusselt number. Further, $R e_{x}=x \sqrt{\frac{a}{v}}$ in the above Eqs. (23) and (24) denotes the local Reynolds number.

\section{Solution methodology}

A two-dimensional magnetized Williamson fluid flow about a sensor surface with variable thermal conductivity and exterior squeezing with viscous dissipation effect gives the highly complicated nonlinear system of flow equations and which are reduced to a simplified form by imposing suitable similarity transformations with appropriate initial boundary conditions. Since, analytical methods fails solve the Eqs. (13) and (14) with relevant boundary conditions Eq. (15). Hence, the present problem is solved with the help of efficient RK-4 technique [51-56]. Further, the considered numerical scheme begun by converting the higher order ordinary differential Eqs. (17) and (18) into the set of first order ordinary differential equations. Also, a care has been taken to choose the boundary of the flow region. However, we taken $\eta=4$ be the boundary and which is considerably far from momentum and thermal boundary layers corresponding to $\eta_{\infty}$. Additionally, it is assumed that, the value of $\eta=4$ is suitable to determine the boundary layer behaviour of different control parameters and which is described in Table 1. Finally, the reduced first order ordinary differential equations corresponding to Eq. (17) are listed below and which are used in the present numerical simulations.

$$
\left.\begin{array}{l}
f(\eta)=\Omega_{1} \\
f^{\prime}(\eta)=\Omega_{1}^{\prime}=\Omega_{2} \\
f^{\prime \prime}(\eta)=\Omega_{2}^{\prime}=\Omega_{3} \\
f^{\prime \prime \prime}(\eta)=\Omega_{3}^{\prime}=\Omega_{4}
\end{array}\right\}
$$

In the above Eq. (25) the value of $\Omega_{4}$ is obtained through the Eq. (17) as follows:

$$
\begin{aligned}
& \Omega_{4}=\left(\frac{1}{1+2 W e \Omega_{3}}\right) \\
& {\left[\Omega_{2}^{2}-\left(\Omega_{1}+\frac{b}{2} \eta\right) \Omega_{3}+b\left(1-\Omega_{2}\right)+M\left(\Omega_{2}-1\right)-1\right]}
\end{aligned}
$$

Also, the set first order ordinary differential equations obtained from Eq. (18) are as follows:

$$
\left.\begin{array}{l}
\theta^{\prime}(\eta)=\Omega_{5}^{\prime}=\Omega_{6} \\
\theta^{\prime \prime}(\eta)=\Omega_{6}^{\prime}=\Omega_{7}
\end{array}\right\}
$$


In the above Eq. (27) the value of $\Omega_{7}$ is obtained through the Eq. (18) as follows:

$$
\begin{aligned}
& \Omega_{7}=\left(\frac{1}{1+\epsilon \Omega_{5}}\right)\left[\operatorname{Pr}\left(\Omega_{2}+\frac{b}{2}\right) \Omega_{5}-\operatorname{Pr}\left(\Omega_{1}+\frac{b}{2} \eta\right) \Omega_{6}\right. \\
& \left.-\epsilon \Omega_{6}{ }^{2}-\operatorname{PrEc}\left(\Omega_{3}{ }^{2}+W e \Omega_{3}{ }^{3}\right)\right]
\end{aligned}
$$

Further, the related boundary conditions of the Eqs. (17) and (18) are taken as follows:

$$
\left.\begin{array}{l}
\Omega_{1}(\eta)=-f_{o}, \quad \Omega_{2}(\eta)=0, \quad \Omega_{6}(\eta)=-1 \text { at } \eta=0 \\
\Omega_{2}(\eta)=1, \quad \Omega_{5}(\eta)=0 \text { at } \eta=\infty
\end{array}\right\}
$$

In order to apply Runge-Kutta method to solve these transformed ordinary differential equations Eqs. (25)-(28), five initial conditions are highly essential. But with Eq. (29) it is clear that, only three initial conditions are known at $\eta=0$. However, the other two initial conditions are obtained by the substitute of $\Omega_{2}(\eta) \rightarrow 1, \Omega_{5}(\eta) \rightarrow 0$ at $\eta \rightarrow$ $\infty$. Thus, this substitution is assumed to be $\Omega_{2}(0)=m_{1}$, $\Omega_{5}(0)=m_{2}$. Further, the Newton-Raphson's technique is effectively used to find the appropriate values of $m_{1}$ and $m_{2}$ for the given set of physical parameters and ambient fluid conditions within the boundary layer region. Finally, the obtained values of $m_{1}$ and $m_{2}$ are improved, in order to satisfy the boundary conditions for $\eta \rightarrow \infty$. Accordingly, the transformed initial value problem is simplified by using RK-4 technique. Additionally, $h=0.01$ be the step size and $10^{-5}$ is convergence criteria in the present study.

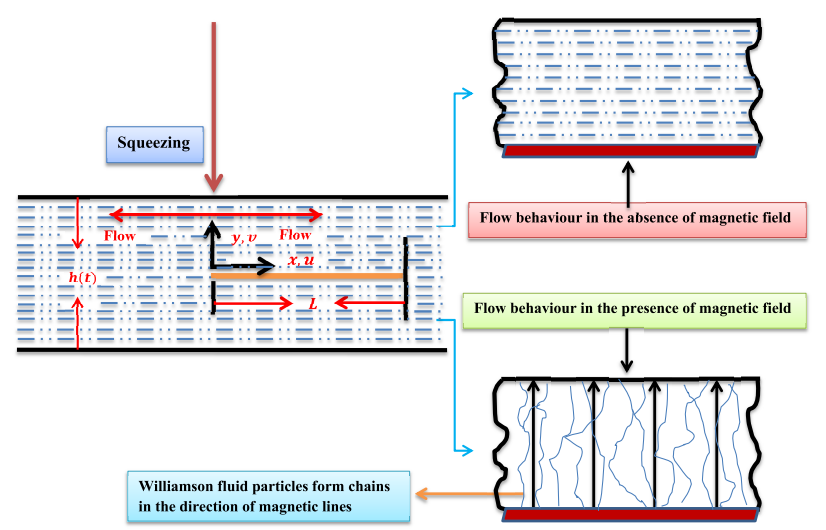

Figure 1: Physical configuration of the present problem

\section{Results analysis}

In this section, the physical behaviour of the various embedding parameters on flow of Williamson fluid over a sen-

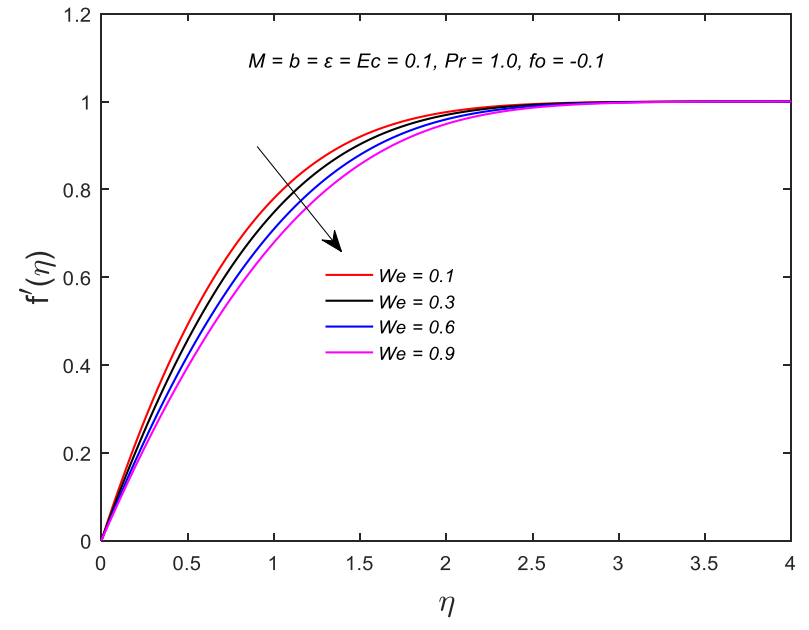

Figure 2: Effect of We on $f^{\prime}(\eta)$

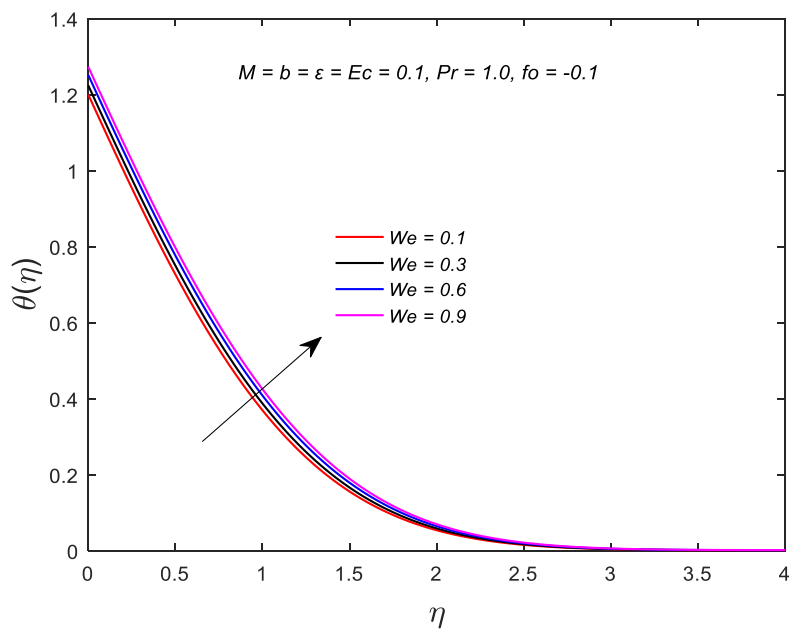

Figure 3: Impact of We on $\theta(\eta)$

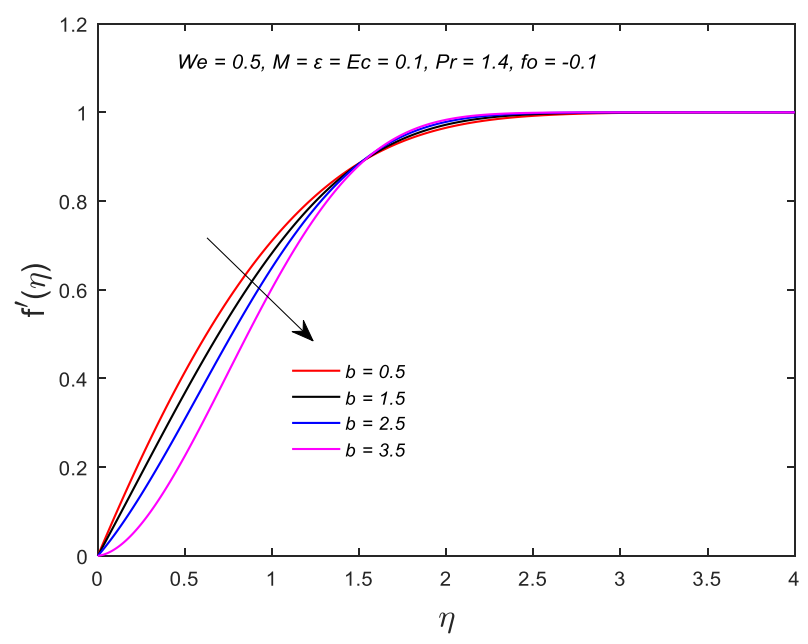

Figure 4: Effect of $b$ on $f^{\prime}(\eta)$ 
Table 1: Computer generated numerical values of momentum transport coefficient for different values of flow parameters

\begin{tabular}{|c|c|c|c|c|c|c|c|}
\hline$W e$ & $b$ & $M$ & $f_{o}$ & $\epsilon$ & $\operatorname{Pr}$ & $E c$ & $f^{\prime \prime}(0)+W e\left(f^{\prime \prime}(0)\right)^{2}$ \\
\hline 0.2 & \multirow{3}{*}{0.1} & \multirow{3}{*}{0.1} & & & & & 1.397482 \\
\hline 0.4 & & & \multirow{2}{*}{-0.1} & \multirow{2}{*}{0.1} & \multirow{2}{*}{1.5} & \multirow{2}{*}{0.1} & 1.473727 \\
\hline 0.6 & & & & & & & 1.539065 \\
\hline 0.8 & 0.0 & & & & & & $\begin{array}{l}1.596947 \\
1.541918\end{array}$ \\
\hline \multirow{25}{*}{0.5} & 0.3 & & & & & & 1.437657 \\
\hline & 0.6 & & & & & & 1.330346 \\
\hline & 0.9 & & & & & & 1.219788 \\
\hline & & 0.2 & & & & & 1.557436 \\
\hline & & 0.4 & & & & & 1.653918 \\
\hline & & 0.6 & & & & & 1.746399 \\
\hline & & 0.8 & & & & & 1.835401 \\
\hline & & & -0.4 & & & & 1.702894 \\
\hline & & & -0.3 & & & & 1.636349 \\
\hline & & & -0.2 & & & & 1.571197 \\
\hline & & & 0.0 & & & & 1.445290 \\
\hline & & & 0.2 & & & & 1.325600 \\
\hline & & & 0.3 & & & & 1.268215 \\
\hline & & & 0.4 & & & & 1.212536 \\
\hline & & & & 0.2 & & & 1.507493 \\
\hline & & & & 0.3 & & & 1.507493 \\
\hline & & & & 0.4 & & & 1.507493 \\
\hline & & & & 0.5 & & & 1.507493 \\
\hline & & & & & 0.5 & & 1.507493 \\
\hline & & & & & 1.0 & & 1.507493 \\
\hline & & & & & 1.5 & & 1.507493 \\
\hline & & & & & 2.0 & 0.2 & $\begin{array}{l}1.507493 \\
1.507493\end{array}$ \\
\hline & & & & & & 0.3 & 1.507493 \\
\hline & & & & & & 0.4 & 1.507493 \\
\hline & & & & & & 0.5 & 1.507493 \\
\hline
\end{tabular}

Table 2: Comparison of present similarity solutions with previously published results of Khaled and Vafai [38] for $\theta(0)$ with $M=f_{o}=\epsilon=0$

\begin{tabular}{cccc}
\hline Prandtl number $(\mathrm{Pr})$ & Squeezed flow $(b)$ index & Khaled and Vafai [38] & Present numerical results \\
\hline 0.71 & & 1.03228 & 1.032282821145898 \\
2.0 & 1.0 & 0.65412 & 0.654123423120187 \\
5.0 & & 0.43561 & 0.435614607270683 \\
6.7 & 0.5 & 0.38182 & 0.381823375689146 \\
\hline & 1.0 & 0.46313 & 0.463137508447626 \\
6.7 & 1.5 & 0.38182 & 0.381823375689146 \\
& 2.0 & 0.33084 & 0.330840498714310 \\
& & 0.29544 & 0.295440261684154 \\
\hline
\end{tabular}

sor surface under squeezing condition is analyzed in view of velocity and temperature profiles, momentum transport coefficient and Nusselt number. Further, the influence of Weissenberg number $(W e)$, squeezed flow index $(b)$, permeable velocity parameter $\left(f_{o}\right)$, magnetic number $(M)$, small parameter $(\epsilon)$, Eckert number $(E c)$ and Prandtl number $(P r)$ on velocity and temperature profiles are described in the Figures 2-12. Also, the variations noticed in momentum and heat transfer rates within the region study for various values of flow parameters are shown in the Table 1 and
Figures 13-15. Further, Table 2 provides the validation of the present numerical solutions.

(i) Impact of Weissenberg number $(W e)$ on $f^{\prime}(\eta)$ and $\theta(\eta)$

The influence of Weissenberg number on velocity and temperature fields are described in the Figures 2 and 3. Figure 2 portrays that, the axial velocity profile diminished with rising values of Weissenberg number. Physically Weissenberg number is the ratio of relaxation time to the specific process time. Here, the magnifying values of Weissenberg number amplify the fluid relaxation time 


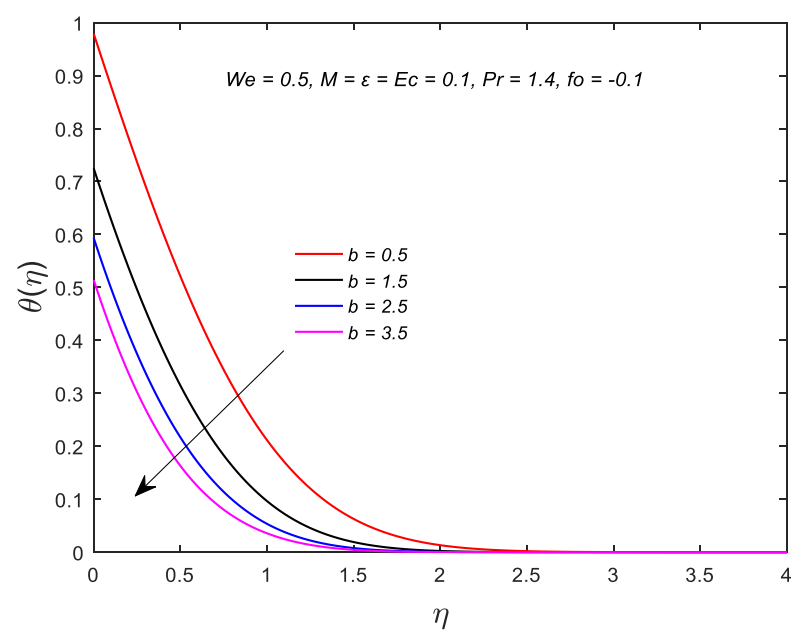

Figure 5: Impact of $b$ on $\theta(\eta)$

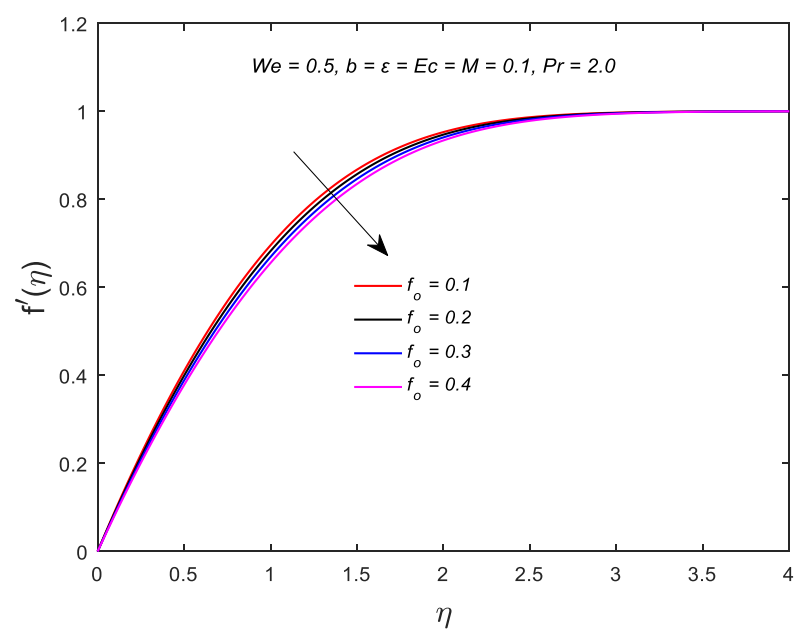

Figure 6: Effect of $f_{o}$ on $f^{\prime}(\eta)$

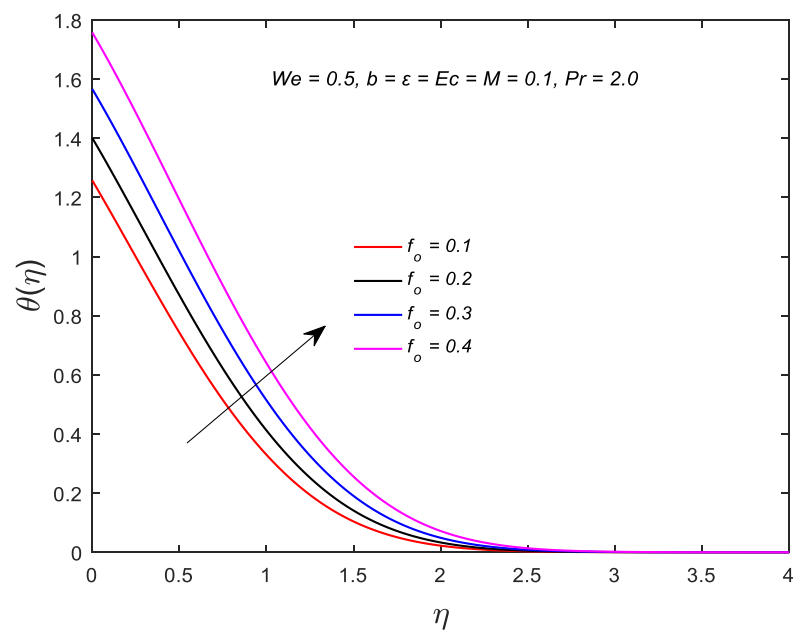

Figure 7: Impact of $f_{o}$ on $\theta(\eta)$

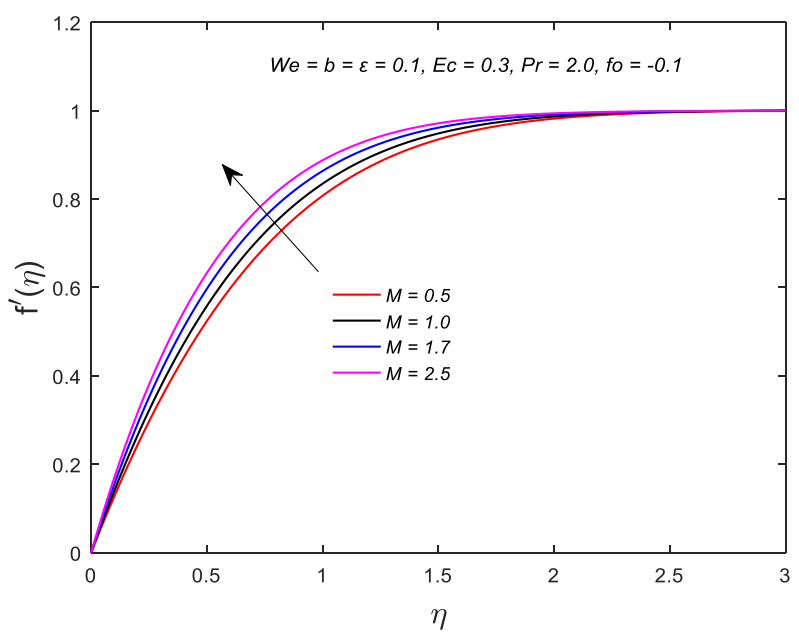

Figure 8: Effect of $M$ on $f^{\prime}(\eta)$

which in turn enhances the resistance to the Williamson fluid flow about sensor surface. Thus, this condition leads to magnification of resistance in the flow region and resulting in a velocity drop as described in the Figure 2. Further, the impact of Weissenberg number on thermal profile is illustrated in the Figure 3. It is observed from Figure 3 that, the temperature field enhanced with rising values of Weissenberg number and which in turn enhance the thermal boundary layer thickness in the flow region. Thus, the temperature profile is an increasing function of Weissenberg number.

(ii) Influence of squeezed flow index $(b)$ on $f^{\prime}(\eta)$ and $\theta(\eta)$

Figures 4 and 5 describe the impact of $b$ on velocity and thermal fields, respectively. However, Figure 4 portrays that rising values of squeezed flow index diminish the velocity field. This decay in velocity profile is owing to the fact that, an increment in squeezing process magnifies the motion of Williamson fluid molecules in the flow direction. However, it is noticed that, there is a reverse relation between squeezed flow index and strength of squeeze flow. Thus, this condition gives the diminished flow velocity with enhancing $b$. Also, due to the variations at the boundary dual velocity behaviour is observed in the channel. Further, Figure 5 describes the impact of $b$ on thermal field in the flow domain. It is important to observe that; the magnified $b$ suppressed the thermal profile. Physically, larger $b$ values reduce the squeezing force on velocity and which in turn diminish the thermal field. It is clear that, the temperature boundary layer thickness is decaying function of squeezed flow index.

(iii) Impact of permeable velocity parameter $\left(f_{o}\right)$ on $f^{\prime}(\eta)$ and $\theta(\eta)$ 


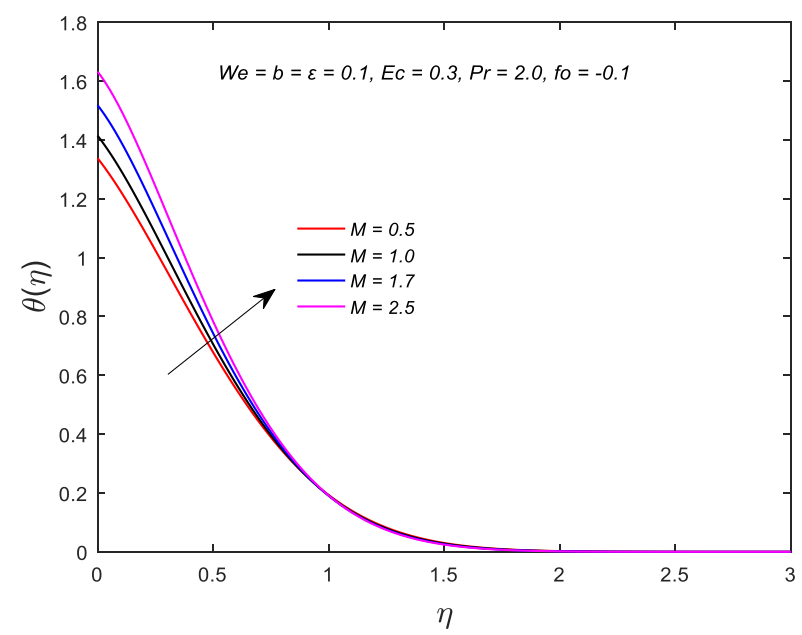

Figure 9: Impact of $M$ on $\theta(\eta)$

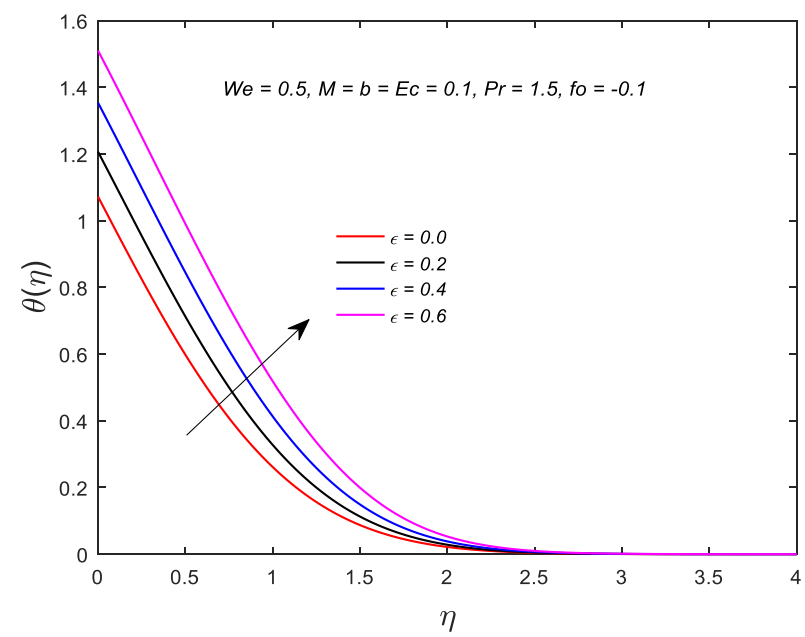

Figure 10: Impact of $\epsilon$ on $\theta(\eta)$

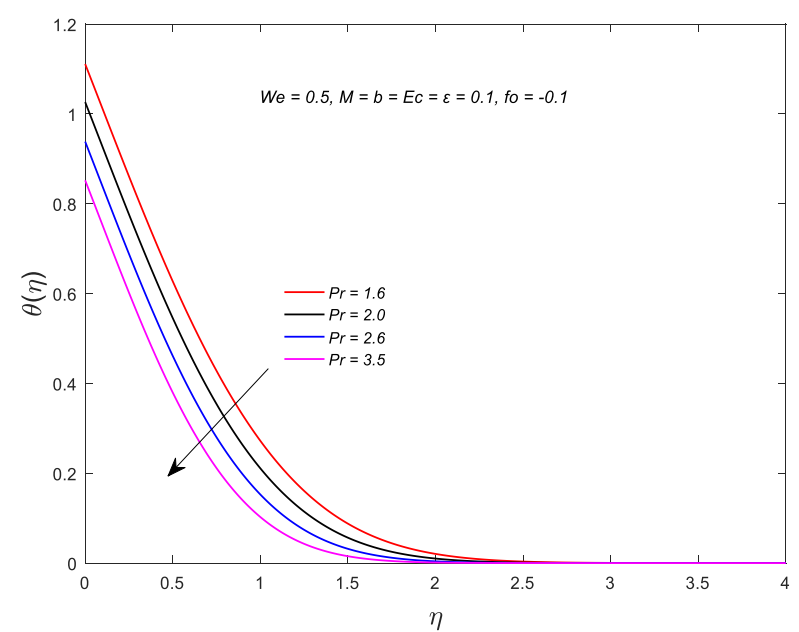

Figure 11: Effect of $\operatorname{Pr}$ on $\theta(\eta)$

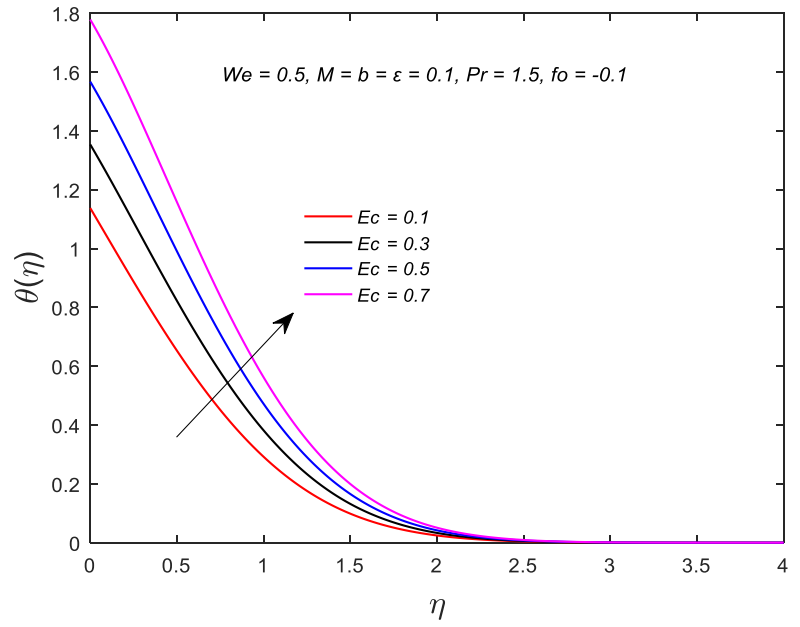

Figure 12: Effect of $E c$ on $\theta(\eta)$

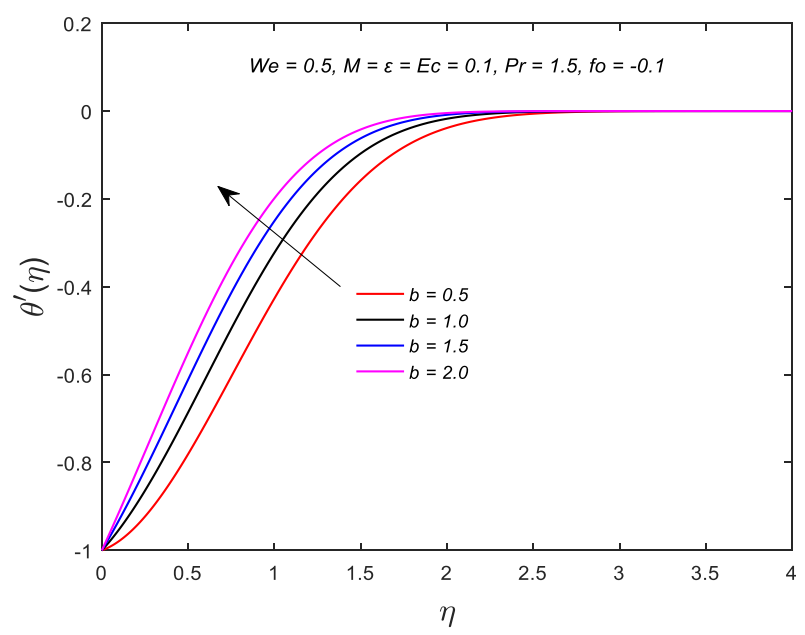

Figure 13: Effect of $b$ on $\theta^{\prime}(\eta)$

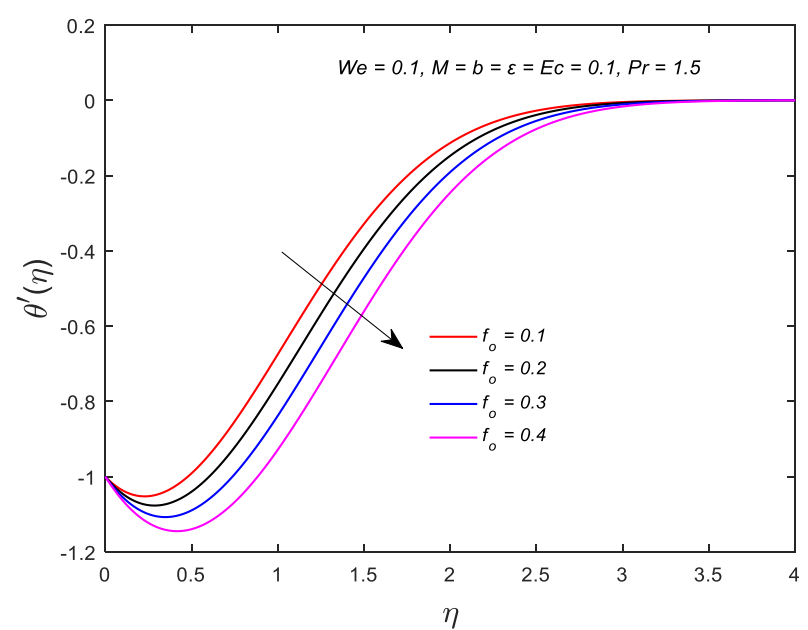

Figure 14: Effect of $f_{o}$ on $\theta^{\prime}(\eta)$ 


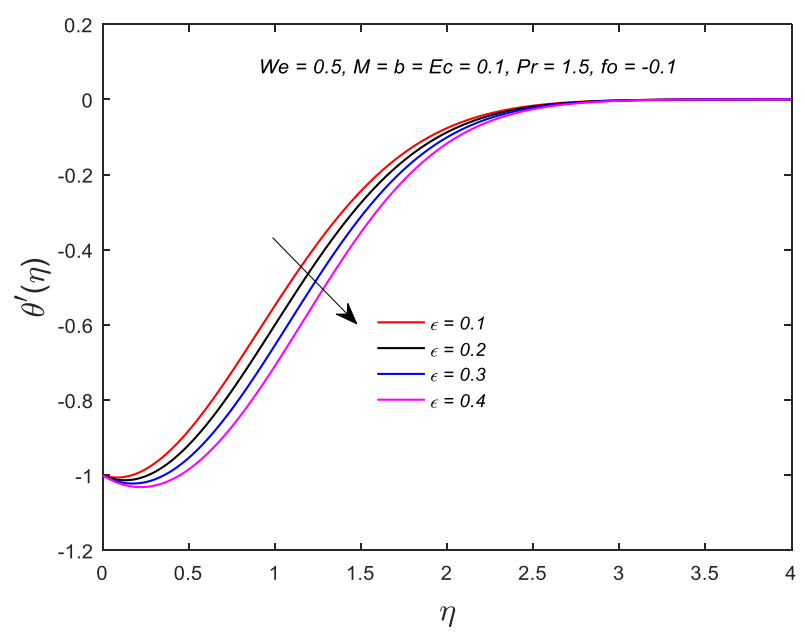

Figure 15: Effect of $\epsilon$ on $\theta^{\prime}(\eta)$

Figures 6 and 7 illustrate the effect of $f_{o}$ on velocity and temperature fields, respectively. It is revealed from Figure 6 that, amplifying $f_{o}$ diminish the velocity field. This decay in axial flow field is because of reason that, for $f_{o}>0$ fluid is largely attached to the sensor surface. This physical condition decays the velocity field inside the channel. Also, momentum boundary layer thickness is a decaying function of permeable velocity parameter. Further, the influence of $f_{o}$ on temperature profile is portrayed in the Figure 7. Figure 7 show that, the increasing permeable velocity parameter enhances the thermal field. This situation describe the cooling of a sensor surface is enhanced under the suction case $\left(f_{o}>0\right)$. Also, the thickness of temperature boundary layer suppressed with rising value of permeable velocity parameter.

(iv) Impact of magnetic parameter $(M)$ on $f^{\prime}(\eta)$ and $\theta(\eta)$

Figures 8 and 9 describe the impact of magnetic parameter on velocity and thermal profiles, respectively. Figure 8 portrays that, the increasing magnetic parameter enhance the velocity profile. Physically, rising magnetic parameter amplifies the resistance along the axial flow direction, since, the upper plate is under squeezing condition, and consequently this physical situation eradicates the impact of applied magnetic field strength on velocity and leading to the enhanced velocity inside the channel. Also, Figure 8 reveals that, the velocity boundary layer thickness enhanced for the increasing values of magnetic number. Figure 9 is devoted to explore the impact of magnetic number on thermal field. It is remarked from Figure 9 that, the enhanced Lorentz forces increases the thermal profile. Also, the thickness of thermal boundary layer enhanced with rising values of magnetic parameter. Thus, temperature field is an increasing function of magnetic parameter.

\section{(v) Effect of small parameter $(\epsilon)$ and Prandtl number $(\operatorname{Pr})$ on $\theta(\eta)$}

Figures 10 and 11 illustrate the thermodynamic behaviour of small parameter $(\epsilon)$ and Prandtl number $(P r)$ on thermal profile, respectively. It is carefully observed from Figure 10 that, the magnifying $\epsilon$ values enhanced the thermal profile. Physically, an upsurge in $\epsilon$ magnifies the molecular motion of liquid molecules and this fact produces the variation in the thermal flow properties of Williamson fluid flow over a sensor surface and consequently the thermal profile increases. Also, it is noticed that, the thickness of temperature boundary layer increases with increment in small parameter. Further, Figure 11 portrays that, the impact of $\operatorname{Pr}$ on thermal field. It is observed from Figure 11 that, the increasing $P r$ decay the thermal profile. This decay in thermal profile is because of the fact that, an upsurge in Pr leads to the diminished temperature diffusivity and hence thermal field suppressed. Also, thickness of temperature boundary layer diminished with rising values of $P r$.

\section{(vi) Effect of Eckert number $(E c)$ on $\theta(\eta)$}

The second term on the right hand side of Eq. (13) represents the viscous dissipation effect which is always positive and indicates a source of heat owing to the frictional forces among the fluid particles. Further, the irreversible process by means of which the work done by a fluid on neighboring fluid layers owing to the action of shear forces is transformed into heat and it is defined as viscous dissipation. Viscous dissipation effect has greater impact in the field of science and engineering such as: polymer processing, injection molding, aerodynamics and etc. However, the boundary layer flows with viscous dissipation effect over sheets/surfaces receives considerable attention in practical applications in a broad spectrum of engineering systems.

The effect of Eckert number $(E c)$ on temperature profile is described in Figure 12. The heat dissipation in the given physical system is primarily calculated in terms of Eckert number. The greater values of Eckert number shows the advective transport process and which generates the considerable effects on heat transfer phenomena as compared to the enthalpy changes in the given physical system or medium. However, it is noticed from Figure 12 that, the thermal profile is an increasing function of Eckert number. This upsurge in temperature profile is obvious because $E c$ has direct impact on the heat dissipation process and which in turn enhances the thermal field. Also, the thermal boundary layer thickness decreases upon the increment in Eckert number. 
(vii) Behaviour of skin-friction coefficient and Nusselt number

The effect of various control parameters on skinfriction coefficient and Nusselt number are illustrated in the Table 1 and Figures 13-15. Table 1 tabulates the computer generated numerical values of skin-friction coefficient for various values of flow parameters. However, it is clear from Table 1 that, the increasing values of $W e$ and $M$ magnifies the skin-friction coefficient whereas enhancing $b$ diminishes the skin-friction coefficient. Further, for $f_{o}<0$ the skin-friction coefficient increases and for $f_{o}>0$ the skin-friction coefficient decreases. Also, the parameters $\epsilon, \operatorname{Pr}$ and $E c$ do not have significant effect on skinfriction coefficient, this is due to their non-explicit occurrence in momentum equation.

Similarly, Figures 13-15 describe the impact of $b, f_{o}$ and $\epsilon$ on heat transfer rate profile. Figures 13-15 begin with negative values because the heat transfer rate formula (refer Eq. (24)) and the temperature boundary condition (refer Eq. (19)) begin with negative sign, due to this reason, the Figures 13-15 begin with negative values. Clearly, Figure 13 illustrates that; the increased squeezed flow index magnifies the heat transfer rate whereas the thermal boundary layer thickness decreases with increasing values of $b$, Physically, increasing squeezed flow index increases the heat transfer rate by producing the high heat molecular forces and high pressure on the fluid flow. Further, it is revealed from Figures 14 and 15 that, the heat transfer rate profile is a decreasing function of permeable velocity parameter and small parameter. Also, the thermal boundary layer thickness increases for the increasing values of $f_{o}$ and $\epsilon$.

\section{(viii) Numerical code testing}

The guarantee and accuracy of present numerical scheme is verified by comparing the current numerical solutions with the results of Khaled and Vafai [38] for $\epsilon=0$. To illustrate this validation, authors have presented Table 2. Table 2 describes that the present similarity results in this article are remarkably corresponding to the results of Khaled and Vafai [38]. However, this authentication endorses the guarantee and accurateness of present similarity solutions. Also, it is noticed from Table 2 that, the temperature field is suppressed for the increasing values of Prandtl number and squeezed flow index.

\section{Concluding remarks}

A two-dimensional magnetized Williamson fluid flow about a sensor surface with variable thermal conductiv- ity and exterior squeezing with viscous dissipation effect is studied numerically. The considered physical problem gives the time-dependent coupled nonlinear partial differential equations and which are reduced to ordinary differential equations by incorporating suitable similarity transformations. The resulting nonlinear flow system is solved by using Runge-Kutta fourth order integration scheme with shooting technique. The physics behind the present problem is dragged out by conducting the parametric study related to the various physical parameters. The graphs and tables are presented to portray the flow sensitivity and thermal behaviour of Williamson fluid about a sensor surface. The important conclusions concerning to the present numerical study is listed below in limiting sense:

- Diminished momentum boundary layer thickness is noticed for the increasing values of Weissenberg number.

- Temperature profile increases for the increasing values of Weissenberg number.

- Velocity and temperature fields suppressed for the increasing squeezed flow index.

- Velocity profile diminished with rising values of permeable velocity parameter. But opposite trend is observed for temperature profile.

- Thinner thermal boundary layer is noticed for increasing magnetic parameter and Prandtl number but the reverse behaviour is true for small parameter $(\epsilon)$.

- Thermal profile increased with increasing values of Eckert number.

- Momentum transport coefficient enhanced with rising values of Weissenberg number and magnetic parameter. But opposite trend is true for increased squeezed flow index.

- Magnitude of Nusselt number increases with rising values of $\epsilon$ and $f_{o}$.

- Runge-Kutta scheme with shooting technique is effective to solve the flow problems arising in the field of science and engineering.

Further, it is anticipated that, the present numerical study is applicable to many of the industrial uses comprising oil recover, thermal flow in fluidic cells, solar collectors and so on.

Acknowledgements: The authors would like to thank the reviewers for their constructive suggestions which led to a definite improvement of the quality of the manuscript. One of the author, Usha Shankar wishes to thank Karnataka Power Corporation limited, Raichur Thermal 
Power Station, Shaktinagar, for their encouragement.

Conflict of interest: The authors declare no conflict of interest regarding the publication of this paper.

\section{References}

[1] Lawal A, Kalyon DM. Squeezing flow of viscoplastic fluids subject to wall slip. Polym Eng Sci. 1998;38(11):1793-804.

[2] Xu C, Yuan L, Xu Y, Hang W. Squeeze flow of interstitial Herschel-Bulkley fluid between two rigid spheres. Particuology. 2010;8(4):360-4.

[3] Engmann J, Servais C, Burbidge AS. Squeeze flow theory and applications to rheometry: A review. J Non-Newt Fluid Mech. 2005;132(1-3):1-27.

[4] Zhang X, Ju H, Wang J. Electrochemical sensors, biosensors and their biomedical applications. USA: Academic Press; 2008.

[5] Lavrik NV, Tipple CA, Sepaniak MJ, Datskos D. Gold-nano structures for transduction of Biomolecular interactions into micrometer scale movements. Biomed Microdevices. 2001;3(1):35-44.

[6] Stefan MJ. Versuchüber die scheinbare adhesion, SitzungsberSächsAkadWissWein, Math-Nat. Wiss. Kl. 1874;69:713-21.

[7] Kuzma DC. Fluid inertia effects in squeeze films. Appl Sci Res. 1968;18(1):15-20.

[8] Gupta PS, Gupta AS. Squeezing flow between parallel plates. Wear. 1977;45(2):177-85.

[9] Wang CY, Watson LT. Squeezing of a viscous fluid between elliptic plates. Appl Sci Res. 1979;35(2-3):195-207.

[10] Usha R, Sridharan R. Arbitrary squeezing of a viscous fluid between elliptic plates. Fluid Dyn Res. 1996;18(1):35-51.

[11] Bhattacharyya S, Pal A. Unsteady MHD squeezing flow between two parallel rotating Discs. Mech Res Commun. 1997;24(6):615-23.

[12] Siddiqui AM, Irum S, Ansari AR. Unsteady squeezing flow of a viscous MHD fluid between parallel plates, a solution using the homotopy perturbation method. Math Model Anal. 2008;13(4):565-76.

[13] Rashidi MM, Siddiqui AM, Rastegari MT. Analytical solution of squeezing flow between two circular plates. Int J Comput Methods Eng Sci Mech. 2012;13(5):342-9.

[14] Mustafa M, Hayat T, Obaidat S. On heat and mass transfer in the unsteady squeezing flow between parallel plates. Meccanica. 2012;47(7):1581-9.

[15] Dogonchi AS, Ganji DD. Study of nanofluid flow and heat transfer between non-parallel stretching walls considering Brownian motion. J Taiwan Inst Chem Eng. 2016;69:1-13.

[16] Hayat T, Sajjad R, Alsaedi A, Muhammad T, Ellahi R. On squeezed flow of couple stress nanofluid between two parallel plates. Results Phys. 2017;7:553-61.

[17] Hayat T, Muhammad T, Qayyum A, Alsaedi A, Mustafa M. On squeezing flow of nanofluid in the presence of magnetic field effects. J Mol Liq. 2016;213:179-85.

[18] Petrov AG, Kharlamova IS. The solutions of Navier-Stokes equations in squeezing flow between parallel plates. Eur J Mech BFluids. 2014;48:40-8.
[19] Hashmi MM, Hayat T, Alsaedi A. On the analytic solutions for squeezing flow of nanofluid between parallel disks. Nonlinear Anal (Vilnius). 2012;17(4):418-30.

[20] Zeeshan A, Baig M, Ellahi R, Hayat T. Flow of viscous nanofluid between the concentric cylinders. J Comput Theor Nanosci. 2014;11(3):646-54.

[21] Hayat T, Yousaf A, Mustafa M, Obaidat S. MHD squeezing flow of second-grade fluid between two parallel disks. Int J Numer Methods Fluids. 2012;69(2):399-410.

[22] Maki ER, Kuzma DC, Donnelly RJ. Magneto-hydrodynamic lubrication flow between parallel plates. J Fluid Mech. 1966;26(3):537-43.

[23] Hayat T, Imtiaz M, Alsaedi A. Impact of magnetohydrodynamics in bidirectional flow of nanofluid subject to second order slip velocity and homogeneous-heterogeneous reactions. J Magn Magn Mater. 2015;395:294-302.

[24] Khan X, Ahmed N, Zaidi ZA, Asadullah M, Mohyud-Din ST. MHD squeezing flow between two infinite plates. Ain Shams Eng J. 2014;5(1):187-92.

[25] Khan M, Qayyum M, Khan O, Ali M. Unsteady squeezing flow of Casson fluid with magneto-hydrodynamic effect and passing through porous medium. Math Probl Eng. 2016;2016:1-14.

[26] Shah RA, Anjum MN, Khan MS. Analysis of unsteady squeezing flow between two porous plates with variable magnetic field. Int. J. Adv. Eng. Mag. Scie. 2017;3(1):90-106.

[27] Uddin MJ, Beg AO, Amin N. Hydromagnetic transport phenomena from a stretching or shrinking nonlinear nanomaterial sheet with Navier slip and convective heating: a model for bio-nano-materials processing. J Magn Magn Mater. 2014;368:252-61.

[28] Hayat T, Nisar Z, Ahmad B, Yasmin HA. Simultaneous effects of slip and wall properties on MHD peristaltic motion of nanofluid with Joule heating. J Magn Magn Mater. 2015;395:48-58.

[29] Dogonchi AS, Ganji DD. Impact of Cattaneo-Christov heat flux on MHD nanofluid flow and heat transfer between parallel plates considering thermal radiation effect. J Taiwan Inst Chem Eng. 2017;80:52-63.

[30] Akmal N, Sagheer M, Hussain S. Numerical study focusing on the entropy analysis of MHD squeezing flow of a nanofluid model using Cattaneo-Christov theory. AIP Adv. 2018;8(5):055201-17.

[31] Malik MY, Hussain A, Salahuddin T, Awais M. Effects of viscous dissipation on MHD boundary layer flow of Sisko fluid over a stretching cylinder. AIP Adv. 2016;6(3):035009-12.

[32] Hayat T, Ullah I, Alsaedi A, Farooq M. MHD flow of PowellEyring nanofluid over a non-linear stretching sheet with variable thickness. Results Phys. 2017;7:189-96.

[33] Mukhopadhyay S. Slip effects on MHD boundary layer flow over an exponentially stretching sheet with suction/blowing and thermal radiation. Ain Shams Eng J. 2013;4(3):485-91.

[34] Ibrahim FN, Terbeche M. Solutions of the laminar boundary layer equations for a conducting power law non-Newtonian fluid in a transverse magnetic field. J Phys D Appl Phys. 1994;27(4):740-7.

[35] Watanabe T, Pop I. Thermal boundary layers in magnetohydrodynamic flow over a flat plate in the presence of a transverse magnetic field. Acta Mech. 1994;105(1-4):233-8.

[36] Ali R, Shahzad A, Khan M, Ayub M. Analytic and numerical solutions for axisymmetric flow with partial slip. Eng Comput. 
2016;32(1):149-54.

[37] Khaled AR, Vafai K. Heat transfer and hydromagnetic control of flow exit conditions inside oscillatory squeezed thin films. Numer Heat Transf A. 2003;43(3):239-58.

[38] Khaled AR, Vafai K. Hydromagnetic squeezed flow and heat transfer over a sensor surface. Int J Eng Sci. 2004;42(56):509-19.

[39] Ul Haq RU, Nadeem S, Khan ZH, Noor NF. MHD squeezed flow of water functionalized metallic nanoparticles over a sensor surface. Physica E. 2015;73:45-53.

[40] Khan M, Malik MY, Salahuddin T, Khan I. Heat transfer squeezed flow of Carreau fluid over a sensor surface with variable thermal conductivity: A numerical study. Results Phys. 2016;6:940-5.

[41] Salahuddin T, Malik MY, Hussain A, Bilal S, Awais M, Khan I. MHD squeezed flow of Carreau-Yasuda fluid over a sensor surface. Alex Eng J. 2017;56(1):27-34.

[42] Kumar KG, Gireesha BJ, Krishanamurthy MR, Rudraswamy NG. An unsteady squeezed flow of a tangent hyperbolic fluid over a sensor surface in the presence of variable thermal conductivity. Results Phys. 2017;7:3031-6.

[43] Ahmad S., Nadeem S., Muhammad N., Khan M.N., Cattaneo-Christov heat flux model for stagnation point flow of micropolar nanofluid toward a nonlinear stretching surface with slip effects, J. Therm. An. Calorim., 2020, DOI: https://doi.org/10.1007/s10973-020-09504-2

[44] Ramesh GK, Gireesha BJ, Gorla RS. Study on Sakiadis and Blasius flows of Williamson fluid with convective boundary condition. Nonlinear Eng. 2015;4(4):215-21.

[45] Shankar U, Naduvinamani NB, Basha H. A generalized perspective of Fourier and Fick's laws: magnetized effects of Cattaneo-Christov models on transient nanofluid flow between two parallel plates with Brownian motion and thermophoresis. Nonlinear Eng. 2020;9(1):201-22.

[46] Mishra SR, Baag S, Dash GC, Acharya MR. Numerical approach to MHD flow of power-law fluid on a stretching sheet with nonuniform heat source. Nonlinear Eng. 2019;9(1):81-93.
[47] Hayat T, Saeed Y, Asad S, Alsaedi A. Soret and Dufour effects in the flow of Williamson fluid over an unsteady stretching surface with thermal radiation. Zeitschrift für Naturforschung. 2015;70(4):235-43.

[48] Hayat T, Shafiq A, Alsaedi A. Hydromagnetic boundary layer flow of Williamson fluid in the presence of thermal radiation and Ohmic dissipation. Alex Eng J. 2016;55(3):2229-40.

[49] Nadeem S, Hussain ST, Lee C. Flow of a Williamson fluid over a stretching sheet. Braz J Chem Eng. 2013;30(3):619-25.

[50] Khan NA, Khan H. A Boundary layer flows of non-Newtonian Williamson fluid. Nonlinear Eng. 2014;3(2):107-15.

[51] Basha H. A generalized perspective of magnetized radiative squeezed flow of viscous fluid between two parallel disks with suction and blowing. Heat Transf Asian Res. 2020;49(4):2248-81.

[52] Shankar U, Naduvinamani NB. Magnetized impacts of Cattaneo-Christov double diffusion models on the timedependent squeezing flow of Casson fluid: A generalized perspective of Fourier and Fick's laws. Eur Phys J Plus. 2019;134:1-24.

[53] Naduvinamani NB, Shankar U. Radiative squeezing flow of unsteady magneto-hydrodynamic Casson fluid between two parallel plates. J Cent South Univ. 2019;26(5):1184-204.

[54] Naduvinamani NB, Shankar U. Thermal-diffusion and diffusion-thermo effects on squeezing flow of unsteady magneto-hydrodynamic Casson fluid between two parallel plates with thermal radiation. Sadhana. 2019;44(8):175-16.

[55] Shankar U, Naduvinamani NB. Magnetized impacts of Brownian motion and thermophoresis on unsteady squeezing flow of nanofluid between two parallel plates with chemical reaction and Joule heating. Heat Transf Asian Res. 2019;48(8):4174-202.

[56] Cebeci T., Bradshaw P., Physical and computational aspects of convective heat transfer, New York, USA: Springer-Verlag; 1984. 